\title{
3D Pose Refinement from Reflections *
}

\author{
Pascal Lagger, Mathieu Salzmann, Vincent Lepetit, Pascal Fua \\ Computer Vision Laboratory \\ EPFL, Switzerland \\ \{pascal.lagger, mathieu.salzmann, vincent.lepetit, pascal.fua\}@epfl.ch
}

\begin{abstract}
We demonstrate how to exploit reflections for accurate registration of shiny objects: The lighting environment can be retrieved from the reflections under a distant illumination assumption. Since it remains unchanged when the camera or the object of interest moves, this provides powerful additional constraints that can be incorporated into standard pose estimation algorithms.

The key idea and main contribution of the paper is therefore to show that the registration should also be performed in the lighting environment space, instead of in the image space only. This lets us recover very accurate pose estimates because the specularities are very sensitive to pose changes. An interesting side result is an accurate estimate of the lighting environment.

Furthermore, since the mapping from lighting environment to specularities has no analytical expression for objects represented as $3 D$ meshes, and is not 1-to-1, registering lighting environments is far from trivial. However we propose a general and effective solution. Our approach is demonstrated on both synthetic and real images.
\end{abstract}

\section{Introduction}

It has long been known that specular reflections provide useful shape and pose information [23, 26, 3]. However, practical approaches to using them to retrieve detailed shape information have only been reported recently $[8,4]$. Moreover, to the best of our knowledge, they have not yet been exploited for pose estimation. As depicted by Figure 1, we show that they can indeed be used to increase the accuracy of registration methods. This is in contrast to all current approaches we know of, which treat reflections, and more generally lighting effects, as nuisances to be discounted using either robust statistics or descriptors that are insensitive to their effects $[10,12]$.

More specifically, we represent the light striking the target object by an environment map. It associates 3D directions with the properties of the light-rays coming from these dation.

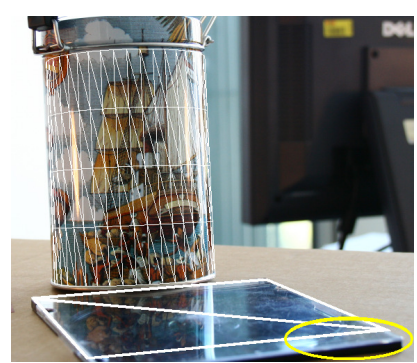

(a) Texture only

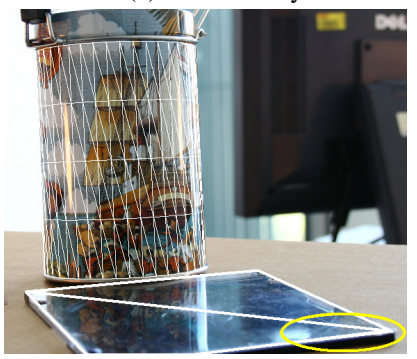

(c) Our method

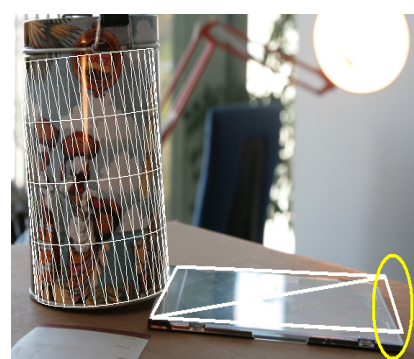

(b) Side-view of (a)

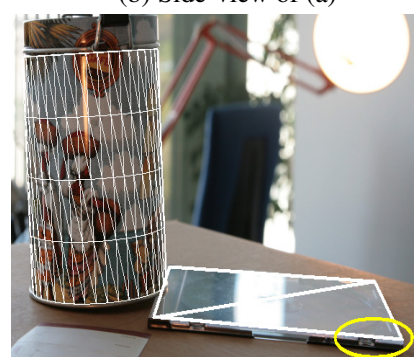

(d) Side-view of (c)
Figure 1. Improving the pose using specular reflection constraints. (a) Projection of a cylindrical model given the pose estimated by standard template matching [2]. The CD case at the bottom side of the image was not used for pose-estimation but we use its known position with respect to the cylinder to project its corresponding two-facet model into the image. The yellow circle highlights the inaccurate reprojection of the case. (b) Projecting the same models in a side-view that was not used to perform pose estimation highlights the inaccuracies, especially at the bottom-right corner of the CD case. (c,d) Projections of the models using the pose obtained by imposing our lighting constraints. They are now much more accurate as evidenced by the side-view.

directions from both primary light sources such as lamps or windows, and secondary ones such as walls. We compute such a map from the specular pixels given a 3D model of the target object and its pose. Our key observation is then that the correct pose parameters are those that predict the correct location for the specular reflections and therefore let us compute environment maps that remain unchanged as the object moves. This strongly constrains the pose parameters because reflections are more sensitive to slight pose changes than texture, as shown in Fig. 2. 


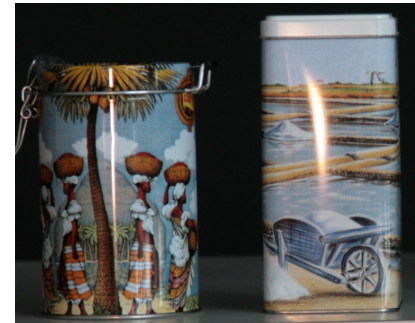

(a)

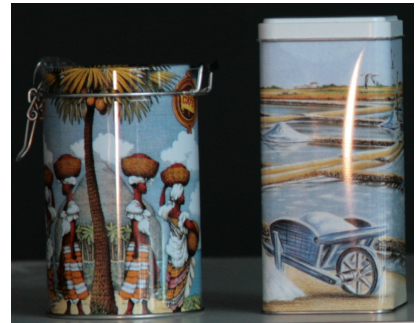

(b)
Figure 2. Specularities provide strong pose constraints. (a) Two shiny boxes. (b) Both boxes have been moved very slightly. The displacement of the specularities is much larger than the motion of the underlying texture and therefore yields much stronger pose constraints.

We show that these lighting constraints can substantially improve the precision of a standard template matching algorithm [2]. For instance, we can reduce by $97 \%$ the residual error of the object to camera distance and horizontal rotation parameters produced when using only template matching. The kind of pose improvement made possible by our method is clearly important in the case of mixed reality, where a small tracking error can result in unrealistic augmented scene, which is what happens to the virtual CD-case of Fig. 1(a,b). We will also show that this is important when trying to re-synthesize specularities at the right places.

Our algorithm can be summarized as follows: Given short image sequences and approximate pose estimates computed by standard template matching, we estimate the Lambertian and specular components for each frame and derive environment maps from the estimated specular images. Since these maps are functions of the pose parameters, we can align them at the same time as we align the image textures and use the same template matching approach to do so.

In short, we simultaneously register in the image space and in the lighting environment space. The main challenge in implementing this approach was to compute environment maps that can be used for this purpose. In particular our maps have to be differentiable with respect to the pose parameters since the registration algorithm requires the analytical computation of derivatives, something that standard approaches to computing environment maps do not provide for. Fig. 3 depicts our solution to this problem, which is very generic. It could be incorporated into other $3 \mathrm{D}$ tracking approaches and thus, open new avenues of research.

\section{Related Work}

Camera pose estimation and 3D tracking have been extensively explored $[10,12]$. They typically involve matching 2D image features against 3D ones and tend to discard lighting information. The use of edge and line features is often justified on the basis of their insensitivity to lighting changes. Most feature-based and template-based ap- proaches rely on normalized cross-correlation or on region descriptors such as SIFT [14] to achieve lighting invariance.

Our approach that explicitly seeks to exploit lighting changes to increase accuracy is therefore more closely related to works on illumination or irradiance recovery, which have long been studied in the Computer Vision and Computer Graphics communities.

In a very generic way, the lighting environment can be formalized by the plenoptic function [1] whose most extensive definition includes light-rays properties for each $3 \mathrm{D}$ position and orientation [7, 9, 13, 6, 25]. However, considering all its degrees of freedom is very cumbersome, and most approaches focus on local regions under the assumption that the rest of the scene is sufficiently far away. That way, the properties of the light-rays depend on their directions alone. This simpler plenoptic function only has 2 degrees of freedom and can be treated as a 2D image, which is often referred to as an environment map.

For some applications, such a map can be built by using fish eye lenses [21] or a panoramic view made from a rotating camera at the center of the region of interest. Another possibility is to use an image acquired using a light probe. For this purpose, a mirrored sphere constitutes an excellent choice both because all directions are represented in its image and because it is easy to map back the environment map using the analytical definition of the sphere [5]. However, it is not always possible to introduce such an ideal light probe in the environment before acquiring the images. To address this issue, the use of random objects that actually are present in the scene has been demonstrated $[17,24]$ or even human eyes [16]. Unlike the sphere mentioned above, such an object may not behave like a perfect mirror and the specular component must first be separated from the Lambertian image texture.

This separation can be achieved in many different ways, depending on the chosen representation for the lighting environment. One may use a sparse representation made of point light sources [18,11], and extract the highlights only as local intensity maxima. However a dense representation is more general. In that case, the specular reflections of the environment on the object surface have to be extracted entirely. In case of dielectric objects and sources of a unique chromaticity, they can be retrieved using [22]. When engineering the capture is possible, polarization approaches such as [15] allow for accurate diffuse-specular separation. [19] considers the image flow, but only in the case of objects that are formed of parts that are either strictly Lambertian or strictly specular. When a 3D model of the object of interest is available, it can be exploited to handle materials with more generic reflection properties [17, 24]. Since the object is represented by a mesh and not as an analytical surface, one has to be careful when mapping back the extracted specularities to the environment map. [17] uses a 


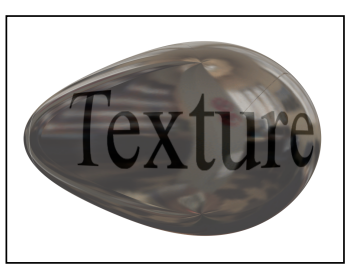

(a)

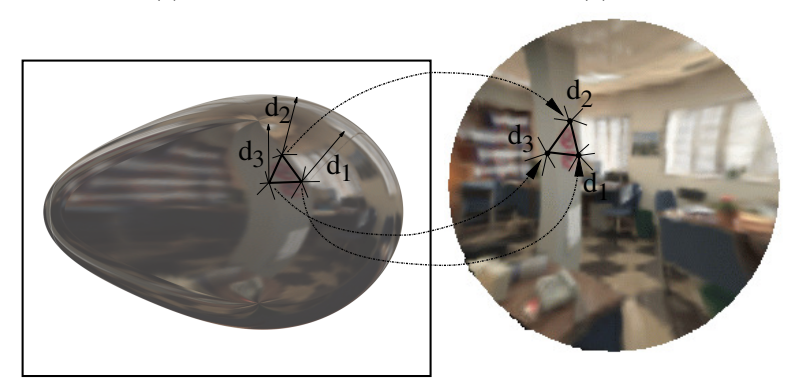

(e)

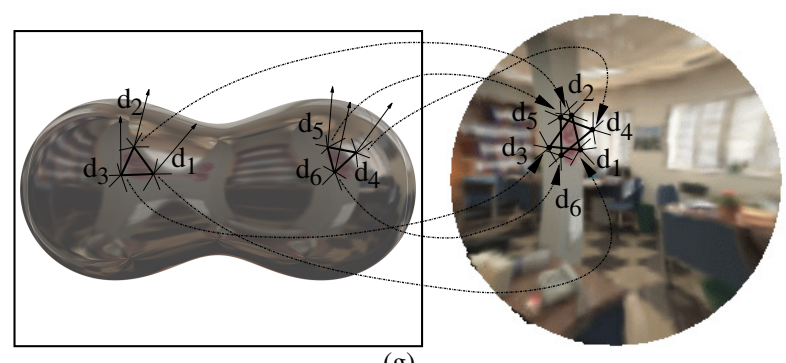

(g)

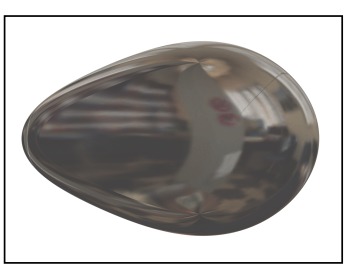

(b) (c)

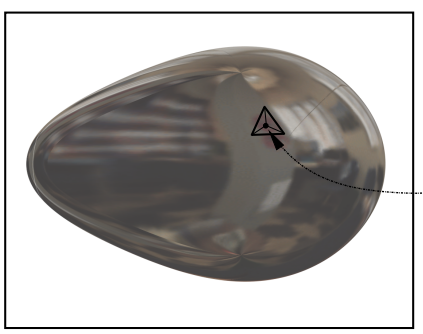

(f)

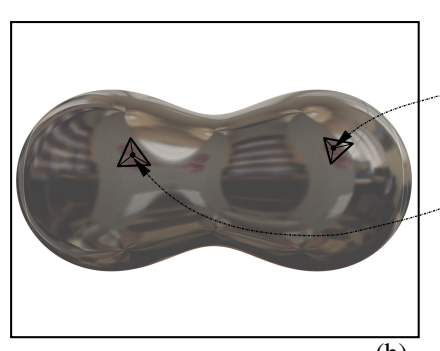

(h)

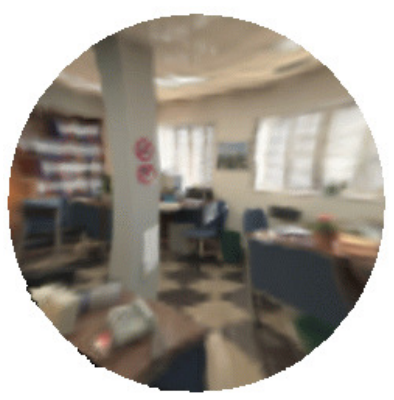

(d)
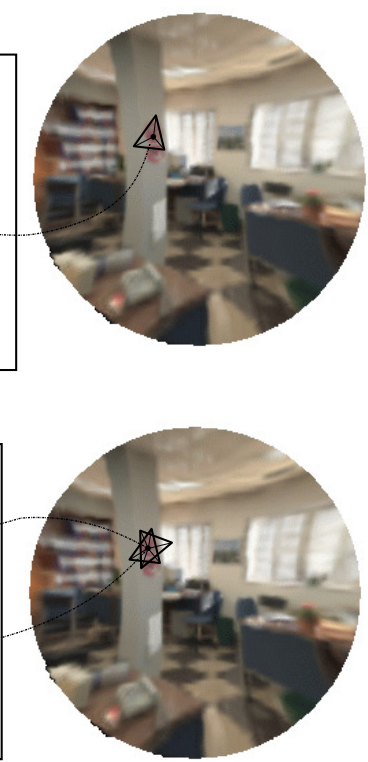

Figure 3. Computing a differentiable environment map from specular image pixels, by associating a color to each 3D orientation. (a) One image from a sequence of a specular and textured objet to which a 3D model is registered. (b) The corresponding specular image. (c) Direct mapping from image to 3D orientation creates holes due to sampling problems and does not allow the computation of the derivatives required for template matching. (d) Inverse mapping produces a map that is suitable for template matching, but cannot be differentiated analytically for objects represented as 3D meshes. (e,f) For convex objects, we overcome this problem by first mapping the mesh vertices from the image to the environment map and then assigning to each environment map point the color obtained by linear interpolation over the triangle the point lies on. (g,h) For non-convex objects, the mapping becomes a many-to-one relation and the final color is taken to be the mean of the colors at locations computed from all the triangles the point lies on.

low resolution environment map called illumination hemisphere and makes each specular pixel vote independently. This gives robustness to pose and reflections extraction errors but results in relatively poor quality maps in which the structure of the specularities is lost. [24] fits a function made of spherical harmonics, and as a result, gets smoothed environment maps. While in their context these approaches to building environment maps are sufficient for shape and texture recovery, we need a more accurate one for precise pose estimation since we match the structure and the shape of the light sources present in the environment.

Our own approach to computing environment maps follows the same philosophy as $[17,24]$ when extracting the specular reflections but is designed to produce sharp and differentiable environment maps, such as those of Fig. 3.

\section{Approach}

Our approach to accurately computing the pose of a target object in each frame of an $n$-image video-sequence $\left\{\mathbf{I}_{i}\right\}_{i=1 . . n}$ includes three steps. Given the pose in the first frame, which can be obtained using a technique based on matching feature points, and an untextured 3D model $\mathcal{M}$ in the form of a triangulated mesh, we first perform standard template matching, which yields rough pose-estimates in the rest of the sequence. From these initial poses, we then separate Lambertian from specular image components. Finally, given the specularities, we refine the pose estimates in the images so that their corresponding environment maps are as similar as possible to the one associated with the first image. In the remainder of this section, we describe these three steps in more detail. 


\subsection{Initial Pose Estimation}

We treat the first image as a reference and perform template matching to estimate the pose in the others. More specifically, given the pose $\boldsymbol{\Theta}_{1}$ in the first image $\mathbf{I}_{1}$, we seek to recover the pose $\Theta_{i}$ in the following ones in terms of 3 exponential map coefficients for rotation and 3 translation parameters. Let $W\left(\mathbf{I}, \boldsymbol{\Theta}, \boldsymbol{\Theta}^{\prime}\right)$ be the function that warps the image of mesh $\mathcal{M}$ with pose $\Theta$ in $\mathbf{I}$ to synthesize the one that would have been produced if its pose had been $\Theta^{\prime}$. We use barycentric coordinates to select regularly spaced $3 \mathrm{D}$ points on each of the $n_{f}$ facets of $\mathcal{M}$ and assign to these points the intensity corresponding to their reprojection in image $\mathbf{I}_{1}$ given pose $\boldsymbol{\Theta}_{1}$. We retrieve the pose $\boldsymbol{\Theta}_{i}$ in image $\mathbf{I}_{i}$ by maximizing

$f_{\mathbf{T}}\left(\boldsymbol{\Theta}_{i}\right)=\sum_{j=1}^{n_{f}} \operatorname{NCC}\left(W\left(\mathbf{I}_{1}, \boldsymbol{\Theta}_{1}, \boldsymbol{\Theta}_{i}\right)\left(\mathbf{p}_{j}\left(\boldsymbol{\Theta}_{i}\right)\right), \mathbf{I}\left(\mathbf{p}_{j}\left(\boldsymbol{\Theta}_{i}\right)\right)\right),(1)$

where the $\mathbf{p}_{j}$ are the $2 \mathrm{D}$ projections of the $3 \mathrm{D}$ points sampled on facet $j$, and $\mathrm{NCC}(.,$.$) represents normalized cross-$ correlation. Bilinear image interpolation lets us treat $f_{\mathbf{T}}$ as a differentiable function of the pose, which is important for optimization purposes.

\subsection{Isolating Specularities}

To extract specularities, we could have used sophisticated methods, such as those proposed [22, 20] for dielectric materials. However, for the sake of both simplicity and generality, we used a much simpler median-based approach, which has proved adequate for our purposes.

Because normalized cross-correlation is relatively insensitive to illumination changes, template matching yields pose estimates that, while not perfectly accurate, are sufficient to estimate the object's underlying texture. As shown by Fig. 4, we estimate the Lambertian component of the texture by warping back all the images to the first one so that corresponding object parts are superposed. In the warped images, the Lambertian image component is stabilized, while the specular one is not. We therefore perform median filtering on a pixel per pixel basis in the stack of images to remove the specular component and represent the object's texture as the image

$$
\mathbf{T}=\operatorname{Median}_{i} W\left(\mathbf{I}_{i}, \boldsymbol{\Theta}_{i}, \boldsymbol{\Theta}_{1}\right) .
$$

The specular component in image $i$ is then taken to be

$$
\mathbf{S}_{i}=\mathbf{I}_{i}-W\left(\mathbf{T}, \boldsymbol{\Theta}_{1}, \boldsymbol{\Theta}_{i}\right) .
$$

$\mathbf{S}_{i}$ is a reliable estimate because the median filtering is robust to the intensity changes produced by small poseestimation inaccuracies. Even though $\mathbf{T}$ is only an approximation of the true object texture when the object moves with respect to the lighting, it suffices for our purposes because specularities typically produce intensity changes much larger than those attributable to Lambertian effects.

\subsection{Computing the Environment Maps}

Given the pose parameters $\Theta$, we represent the environment map as an image $\mathbf{E}(\boldsymbol{\Theta}, \mathbf{S})$ corresponding to a $2 \mathrm{D}$ mapping of a sphere, and whose intensities come from a specular image $\mathbf{S}$. We follow the mapping function of [5] since it results in low distortion in all directions, but extend it to $3 \mathrm{D}$ meshes while making it differentiable.

We build our environment maps as described in Fig. 3(eh): We first project the vertices of our triangulated mesh $\mathcal{M}$ into the environment map $\mathbf{E}$. Each resulting 2D triangle is filled by interpolating the values in the specular image $\mathbf{S}_{i}$. This solves the sampling problem illustrated by Fig. 3(c). However, special care is required to handle the fact that several points in the specular images can fall at the same environment map location because they correspond to the same normals.

More specifically, given the 3D vertices $\left\{\mathbf{v}_{k}\right\}$ of $\mathcal{M}$, we compute the viewing direction $\mathbf{e}_{k}(\boldsymbol{\Theta})$ of vertex $\mathbf{v}_{k}$ as the normalized vector joining it to the camera center. The direction $\mathbf{d}_{k}(\boldsymbol{\Theta})$ of the light-ray striking the object at $\mathbf{v}_{k}$ and reaching the camera is given by

$$
\mathbf{d}_{k}(\boldsymbol{\Theta})=\frac{\mathbf{e}_{k}(\boldsymbol{\Theta})-2\left(\mathbf{e}_{k}(\boldsymbol{\Theta}) \cdot \mathbf{n}_{k}\right) \mathbf{n}_{k}}{\left\|\mathbf{e}_{k}(\boldsymbol{\Theta})-2\left(\mathbf{e}_{k}(\boldsymbol{\Theta}) \cdot \mathbf{n}_{k}\right) \mathbf{n}_{k}\right\|}
$$

where $\mathbf{n}_{k}$ is the normal to the object's surface at vertex $\mathbf{v}_{k}$. Projecting $\mathbf{v}_{k}$ in the environment image then amounts to computing the point $\mathbf{q}_{k}$ in the environment map that corresponds to direction $\mathbf{d}_{k}(\boldsymbol{\Theta})=\left[\mathbf{d}_{x}, \mathbf{d}_{y}, \mathbf{d}_{z}\right]^{\mathrm{T}}$

$$
\mathbf{q}_{k}(\boldsymbol{\Theta})=\left[\begin{array}{l}
0.5+\frac{\mathbf{d}_{x} \arccos \left(\mathbf{d}_{z}\right)}{2 \pi \sqrt{\mathbf{d}_{x}^{2}+\mathbf{d}_{y}^{2}}} \\
0.5+\frac{\mathbf{d}_{y} \arccos \left(\mathbf{d}_{z}\right)}{2 \pi \sqrt{\mathbf{d}_{x}^{2}+\mathbf{d}_{y}^{2}}}
\end{array}\right] .
$$

We then exploit $\mathbf{q}_{k}$ to estimate the intensity at each location $\mathbf{s}$ in $\mathbf{E}$. We assume that the facets of the mesh are sufficiently small so that their projections in $\mathbf{E}$ remain triangles, which can always be achieved by retriangulation of the mesh. At location $\mathbf{s}=[s, t]^{\mathrm{T}}$ in the environment image, the intensity $\mathbf{E}(\boldsymbol{\Theta}, \mathbf{S})(\mathbf{s})$ is taken as the mean of the intensities in $\mathbf{S}$ at location obtained from the facets on which $\mathbf{S}$ lies.

We first compute the barycentric coordinates of $\mathbf{s}$ for every facet $j$ it lies on

$$
\mathbf{b}^{j}(\boldsymbol{\Theta})=\left[\begin{array}{ccc}
p_{1}^{j} & p_{2}^{j} & p_{3}^{j} \\
q_{1}^{j} & q_{2}^{j} & q_{3}^{j} \\
1 & 1 & 1
\end{array}\right]^{-1}(\boldsymbol{\Theta})\left[\begin{array}{l}
s \\
t \\
1
\end{array}\right]
$$

where $p_{k}^{j}$ and $q_{k}^{j}$ are the environment image coordinates of the $k^{t h}$ vertex of facet $j$. The environment image can finally be written as

$$
\mathbf{E}(\boldsymbol{\Theta}, \mathbf{S})(\mathbf{s})=\frac{1}{n_{f}} \sum_{j=1}^{n_{f}} \mathbf{S}\left(\mathbf{m}^{j}(\boldsymbol{\Theta})\right)
$$




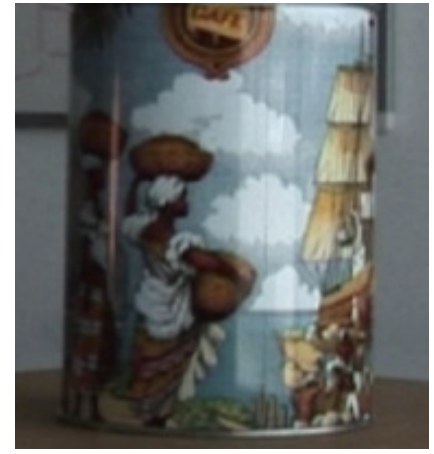

(a)

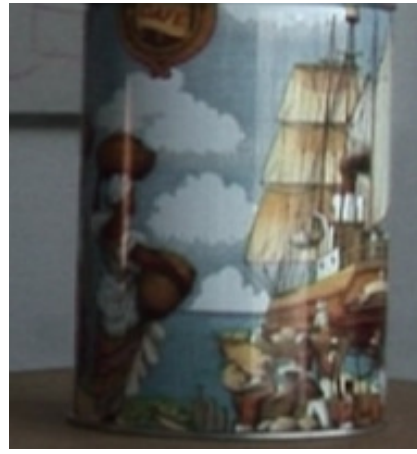

(b)

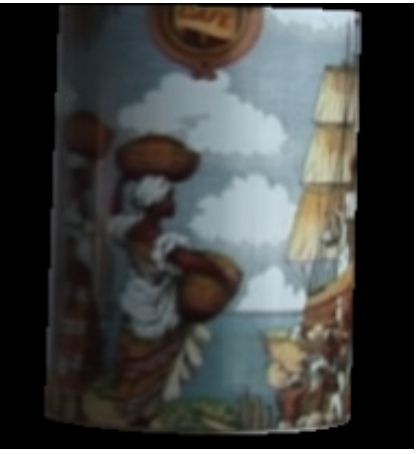

(c)

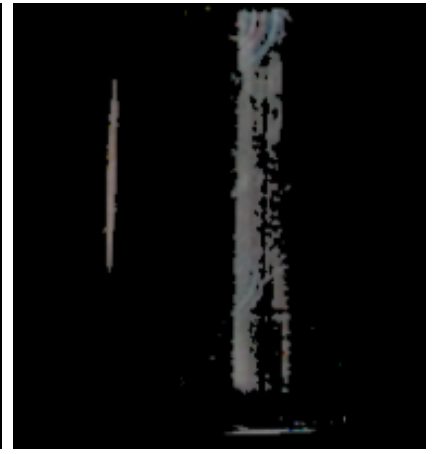

(d)

Figure 4. Estimation of texture and reflections. (a,b) First and last frame of a video sequence. (c,d) The diffuse and specular images computed as described in Section 3.2. Image (d) was gamma-corrected for clarity.

where the $\mathbf{m}^{j}$ are locations in the specular image defined by the previously computed barycentric coordinates $\mathbf{b}^{j}$ with respect to the projection of facet $j$ in $\mathbf{S}$. Note that $\mathbf{E}(\boldsymbol{\Theta}, \mathbf{S})$ is differentiable with respect to $\Theta$, which is critical for template matching.

\subsection{Pose Refinement from Environment Maps}

In each image $\mathbf{I}_{i}$ of the sequence, starting from the rough estimate of the pose $\boldsymbol{\Theta}_{i}$ of Section 3.1 and under the assumption of static lighting, we can now use both the texture and environment map representations to refine the pose by minimizing

$$
\begin{aligned}
f\left(\boldsymbol{\Theta}_{i}\right) & =\min _{\boldsymbol{\Theta}} f_{\mathbf{E}}\left(\boldsymbol{\Theta}_{i}\right)-\lambda f_{\mathbf{T}}\left(\boldsymbol{\Theta}_{i}\right), \text { with } \\
f_{\mathbf{E}}\left(\boldsymbol{\Theta}_{i}\right) & =\sum\left(\mathbf{E}\left(\boldsymbol{\Theta}_{i}, \mathbf{S}_{i}\right)-\mathbf{E}\left(\boldsymbol{\Theta}_{1}, \mathbf{S}_{1}\right)\right)^{2}
\end{aligned}
$$

where $f_{\mathbf{T}}$ is the texture correlation term of Eq. 1, and $f_{\mathbf{E}}$ quantifies the difference between the environment maps estimated from the first frame and from the current one. $\lambda$ is a weight that we compute so that the two terms have gradients with initially equal magnitude, thus ensuring that they have a comparable influence.

Fig. 5 depicts energy landscapes around a correct pose in one frame of a video sequence. To produce them, we have taken a known pose and evaluated both $f_{\mathbf{T}}$ and $f_{\mathbf{E}}$ at various poses around it. To represent it in $2 \mathrm{D}$ we have fixed 4 parameters and sampled the $\mathrm{Z}$-axis translation and the $\mathrm{X}$-axis rotation, which are the two parameters that are only weakly constrained by texture matching in the example of Fig. 1. The lighting energy landscape of Fig. 5(b) shows a steep energy drop at the correct pose for X-axis rotation, which greatly constrains the optimization. This can be seen in the total energy landscape of Fig. 5(c), which exhibits a deeper narrower energy minimum than the one of Fig. 5(a) computed from texture only. This means that the global minimum is defined with more accuracy and that it can be reached more easily. These are the root reasons why combining texture and lighting constraints brings the improvement we demonstrate below.

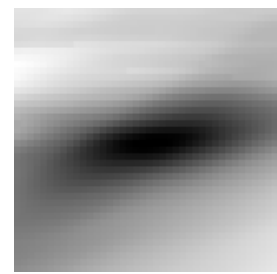

(a)

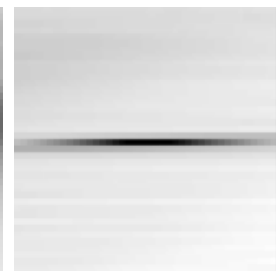

(b)

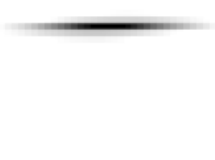

(c)
Figure 5. Energy landscapes with image center corresponding to the correct pose. Each image is a plane cutting the $6 \mathrm{D}$ space of the energy function. When evaluating the energy, we only let the $\mathrm{X}$-axis rotation and the Z-translation vary, since they are the less constrained parameters in a texture-based tracking, and set the others to their optimal values. (a) is the texture energy, (b) the lighting one, and (c) the addition of the two, which exhibits a much better defined minimum that the other two.

\section{Results}

We demonstrate our approach first on synthetic images and then on real ones.

\subsection{Synthetic Images}

Fig. 6 illustrates our results using synthetic images for which we can quantify the improvement in accuracy that the lighting constraints provide. The synthetic poses used to generate the images are randomly perturbed. These poses are then independently refined using either only texture or both texture and environment maps. Table 1 shows the distance between the recovered poses and the original ones. Our approach brings substantial improvements over using texture alone, especially as far as estimating Z-Axis translation and rotation around the $\mathrm{X}$-Axis are concerned. These two parameters are precisely those that are difficult to recover using texture cues only because they can vary a lot more than others without changing much the projected appearance. It is of interest to note that the magnitude of the residual errors of all the parameters optimized with our method are now of the same order, which was not the case 


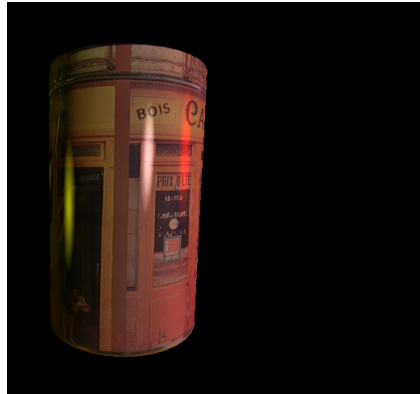

(a) Synthetic image

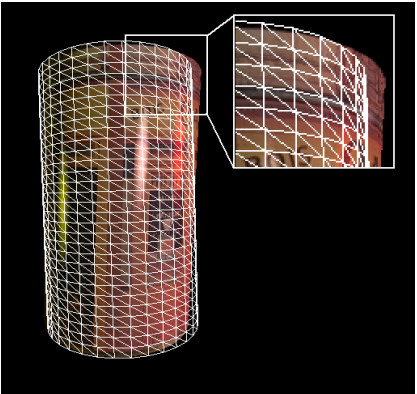

(b) Randomized pose

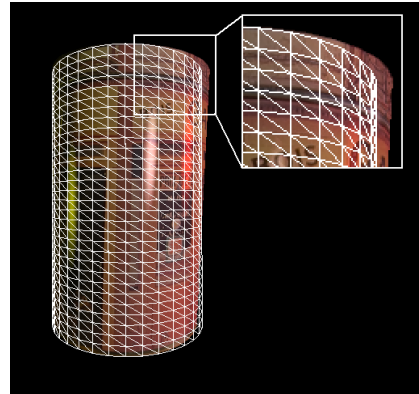

(c) Texture only

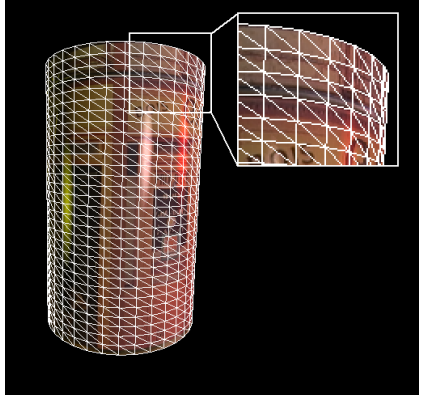

(d) Our approach

Figure 6. Using synthetic images. (a) Original frame from a sequence we synthesized. (b) The pose has been randomized. As a result the top right corner of the cylinder projects at the wrong place. (c) Simple template matching falls into a local minimum and barely improves the pose. (d) Using the lighting constraints brings a substantial improvement and the 3D model now projects at the right place.

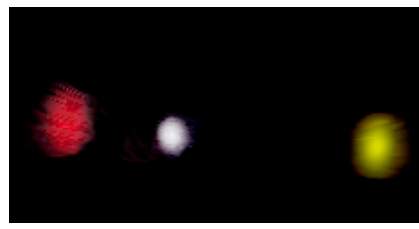

(a) Original environment map

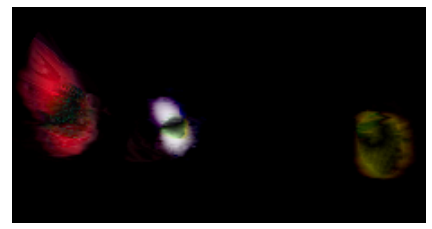

(b) Differences with randomized pose

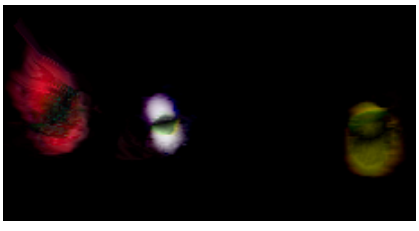

(c) Differences with texture pose

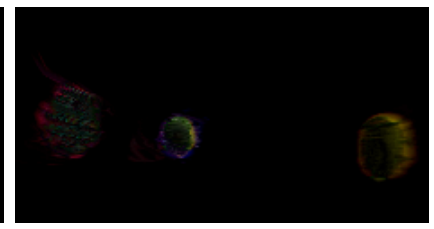

(d) Differences with our method

Figure 7. Partial views of the environment maps for the can of Fig. 6. (a) The original environment map used to produce Fig. 6 (a). (b) The difference image between the original environment map and the one computed by the projection of the specularities present in image Fig. 6(a), using the method of section 3.3(a) and the initial randomized pose. Differences from ground truth mainly involve elongated and skewed lighting areas. (c) Difference image with the environment map obtained after the optimization based on texture cues only. The areas are still skewed and too spread, hence the big differences. (d) By contrast, the difference image obtained after using texture and lighting cues is almost black, which means that the shape and localization of the lighting areas in the recovered environment are very similar to the target image (a). This is related to the accurate pose recovery that is quantified in Table 1 and visible in Fig. 6 (d).

\begin{tabular}{|c|c|c|c|}
\hline $\begin{array}{c}\text { Camera Position } \\
\text { Error in mm on : }\end{array}$ & $\begin{array}{c}\text { Texture } \\
\text { Approach }\end{array}$ & $\begin{array}{c}\text { Our } \\
\text { Method }\end{array}$ & $\begin{array}{c}\text { Error } \\
\text { Reduction }\end{array}$ \\
\hline $\mathrm{x}$ & $1.16(1.35)$ & $0.31(0.33)$ & $73.4 \%$ \\
$\mathrm{y}$ & $1.60(2.79)$ & $0.20(0.19)$ & $87.3 \%$ \\
$\mathrm{z}$ & $\mathbf{7 . 4 2}(\mathbf{9 . 7 0})$ & $\mathbf{0 . 2 0}(\mathbf{0 . 3 3})$ & $97.4 \%$ \\
\hline \hline Camera Rotation & Texture & Our & Error \\
Error in Deg. on : & Approach & Method & Reduction \\
\hline $\mathrm{x}$ & $\mathbf{2 . 4 2}(\mathbf{3 . 1 7})$ & $\mathbf{0 . 0 8}(\mathbf{0 . 1 3})$ & $96.7 \%$ \\
$\mathrm{y}$ & $0.29(0.51)$ & $0.14(0.14)$ & $54.1 \%$ \\
$\mathrm{z}$ & $0.78(1.10)$ & $0.06(0.09)$ & $94.2 \%$ \\
\hline
\end{tabular}

Table 1. Error evaluation in synthetic images, average values (Standard deviation). The Z-axis translation indetermination and $\mathrm{X}$-axis rotation indetermination are resolved by the lighting term. For these parameters, our method reduces the residual error by about $97 \%$.

with the texture only approach. This emphasizes the idea that lighting and texture clues are complementary.

In Fig. 7, we compare the environment map we recover with the one that was used to create the images. They are very similar, which means that our recovered map could be used to convincingly light virtual objects added to the real scene. This was not the case with the environment that can be estimated using the poses from simple texture matching.

\subsection{Real Images}

Fig. 8 shows an example in which combining texture and lighting constraints help. The duck is not very textured. As a result, when using texture only, the recovered poses yield correct reprojection in the middle of the duck - where the texture is - but not at the edges as shown in Fig. 8(b,c). Using the lighting constraints fixes this problem and results in a correct reprojection everywhere. In particular, as shown on fig. 8(d,e), the head and tail now reproject correctly mainly because the $\mathrm{X}$-axis rotation is better constrained.

In Fig. 9, we come back to our central theme. Even the small pose-estimation inaccuracy that results from using only the texture and ignoring illumination effects results in a dramatic motion of the specularities when using this pose to synthesize new images. This motion disappears when using the poses returned by our algorithm.

Accurate pose estimation is crucial for augmented and mixed-reality. In Fig. 1 we demonstrate that our method can refine poses up to a point where it becomes possible to add synthetic objects at correct places even if they are relatively far from the observed object. Furthermore, it shows that the accuracy is conserved in a perpendicular view, which is 


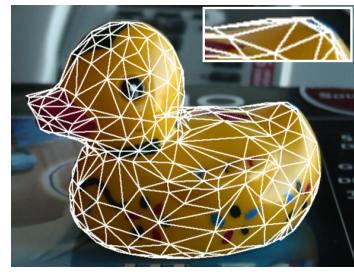

(a) Initial pose

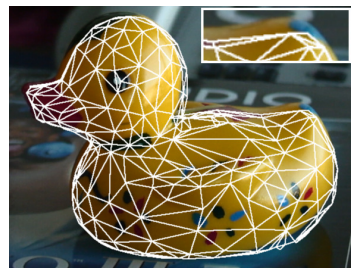

(b) Texture only

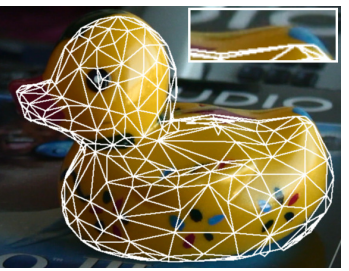

(c) Texture only

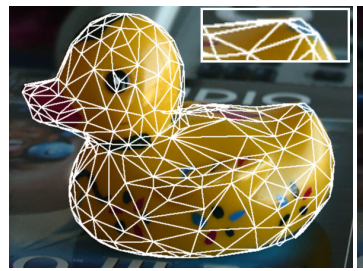

(d) Our approach

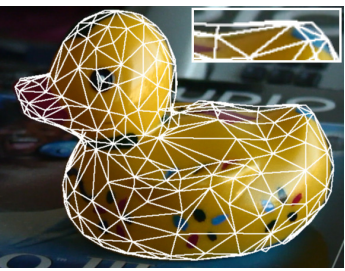

(e) Our approach

Figure 8. Tracking a poorly-textured duck. (a) First frame of the video sequence with registered 3D model. We show in the upper right corner a zoomed version of its tail. $(b, c)$ Using texture only leads to an increasing drift, as evidenced by the fact that the model's occluding contours are not at the right places. $(\mathrm{d}, \mathrm{e})$ The lighting constraints suppress the drift and the model is correctly projected. The corresponding video can be found at http://cvlab.epfl.ch/research/poseref.

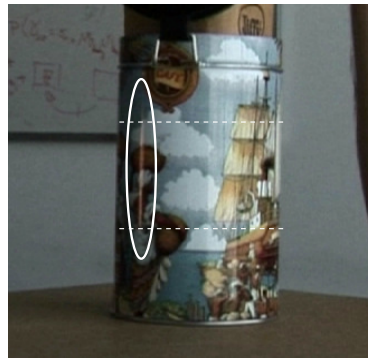

(a)

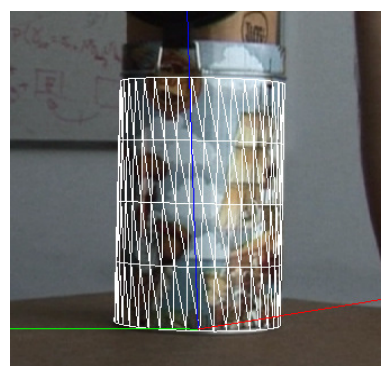

(b)

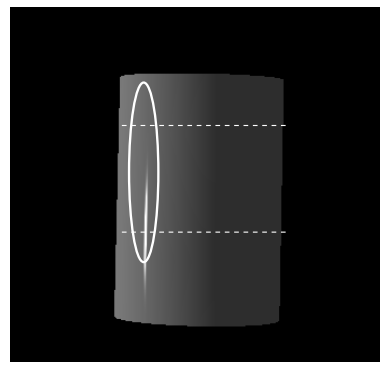

(d)

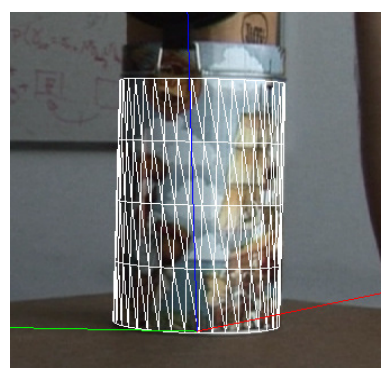

(c)

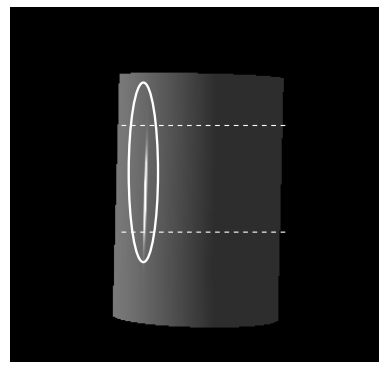

(e)
Figure 9. Resynthesizing specularities. (a) An image with a specularity highlighted by the overlaid ellipse and white lines. It is produced by a point light source at a known location. (b,c) Poses recovered without and with the lighting constraints. (d) Relighting the model using the pose estimated using texture only produces a specularity at the wrong place. (e) Using the lighting constraints yields a refined pose that produces a specularity at the right place.

not used for pose estimation, and where using texture only yields inaccurate results.

Fig. 10 shows the Lambertian appearance of another shiny object and the environment map, both recovered from a 300-frame sequence. Fig. 11 shows that we can use them (a) Initial pose

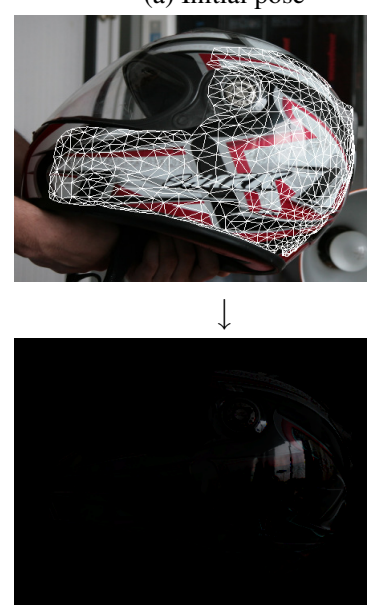

(c) Specular image (b) Texture
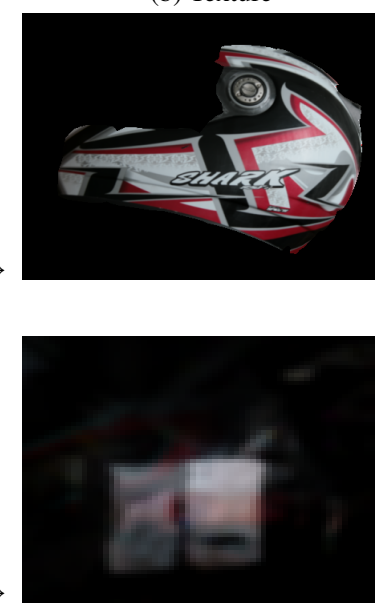

(d) Environment map
Figure 10. Computing an environment map from a 300-frame video. (a) Initial pose of a partial model of the helmet in the first frame of the video. $(b, c)$ Recovered diffuse and specular pixels. (d) Close-up on the computed environment map in which we can see the reflection of an office window.

to synthesize new views in which the reflections are accurately located, which is important for graphics and MixedReality applications.

\section{Conclusion}

Standard pose-estimation algorithms tend to discard lighting-information. In this paper, we have shown that it can be explicitly used to increase the accuracy of a standard template matching algorithm. Our approach relies on a very generic computation of environment maps. It could therefore be incorporated into other 3D tracking approaches, hence opening new avenues of research.

Convincing results were obtained for both accurate object localization in side view and realistic synthetic rendering of shiny objects which are known to require high precision in geometry and pose estimation.

The ability to estimate poses that are both accurate and consistent with a recovered representation of the lighting 

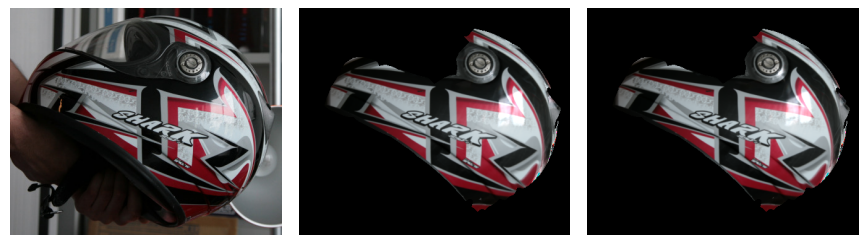

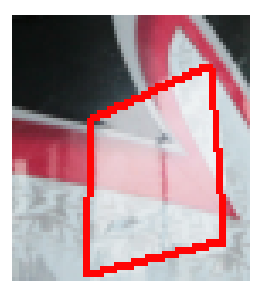

(a) Real image

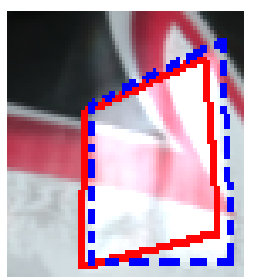

(b) Texture only

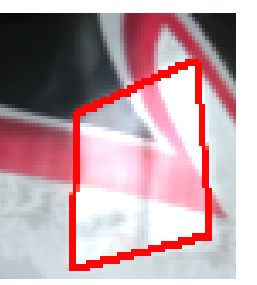

(c) Our approach
Figure 11. Synthesizing images with correctly located and shaped specularities. (a) Top: Frame \#200 in the video sequence of Fig. 10. Bottom: A close-up of this image around the specularity created by the window. We manually outlined it in red. (b) Top: Image synthesized by relighting the recovered texture using the environment map and the pose computed using texture only. Bottom: The corresponding close-up. The red outline is the same as in (a). The blue dotted line delineates the synthesized specularity and does not match the red contour. (c) Top: Synthesizing using the pose computed with our method. Bottom: Note that the rectangular shape of the office window specularity is perfectly clear and exactly matches the red outline.

environment offers interesting opportunities for future applications, such as optimizing the surface normals of the object for refining the shape of the 3D input model and to estimate the reflections properties of the object's material. Mixed-reality applications can also take advantage of the accurate poses and environment estimate.

\section{References}

[1] E. Adelson and J. Bergen. Computational Models for Visual Processing. MIT Press, 1991.

[2] S. Baker and I. Matthews. Lucas-kanade 20 years on: A unifying framework. International Journal of Computer Vision, pages 221-255, March 2004.

[3] G. Brelstaff. Inferring Surface Shape from Specular Reflections. PhD thesis, University of Edinburgh, 1989.

[4] T. Chen, M. Goesele, and H.-P. Seidel. Mesostructure from specularity. In Conference on Computer Vision and Pattern Recognition, pages 1825-1832, 2006.

[5] P. Debevec. A tutorial on image-based lighting. Computer Graphics and Applications, Jan/Feb 2002.

[6] G. Drettakis, L. Robert, and S. Bougnoux. Interactive common illumination for computer augmented reality. In Eurographics, pages 45-57, May 1997.

[7] A. Fournier, A. Gunawan, and C. Romanzin. Common illumination between real and computer generated scenes. In Proceedings of Graphics Interface, pages 254-262, 1993.

[8] A. Gardner, C. Tchou, T. Hawkins, and P. Debevec. Linear light source reflectometry. ACM SIGGRAPH, 2003.
[9] S. Gortler, R. Grzeszczuk, and R. Szeliski. The lumigraph. In ACM SIGGRAPH, pages 43-54, 1996.

[10] R. Hartley and A. Zisserman. Multiple View Geometry in Computer Vision. Cambridge University Press, 2000.

[11] H. Lensch, J. Kautz, M. Goesele, W. Heidrich, and H.-P. Seidel. Image-based reconstruction of spatial appearance and geometric detail. ACM Transactions on Graphics, pages 234-257, 2003.

[12] V. Lepetit and P. Fua. Monocular model-based 3d tracking of rigid objects: A survey. Foundations and Trends in Computer Graphics and Vision, 1(1):1-89, October 2005.

[13] M. Levoy and P. Hanrahan. Light field rendering. In ACM SIGGRAPH, pages 31-42, 1996.

[14] D. Lowe. Distinctive Image Features from Scale-Invariant Keypoints. International Journal of Computer Vision, 20(2):91-110, 2004.

[15] S. K. Nayar, X.-S. Fang, and T. Boult. Separation of reflection components using color and polarization. International Journal of Computer Vision, 21(3):163-186, 1997.

[16] K. Nishino and S. Nayar. Eyes for relighting. ACM Transactions on Graphics, 23(3):704-711, July 2004.

[17] K. Nishino, Z. Zhang, and K. Ikeuchi. Determining Reflectance Parameters and Illumination Distribution from a Sparse Set of Images for View-Dependent Image Synthesis. In International Conference on Computer Vision, pages 599606, July 2001.

[18] M. Powell, S. Sarkar, and D. Goldgof. A simple strategy for calibrating the geometry of light sources. IEEE Transactions on Pattern Analysis and Machine Intelligence, 23:1022-1027, 2001.

[19] S. Roth and M. Black. Specular Flow and the Recovery of Surface Structure. In Conference on Computer Vision and Pattern Recognition, New York, 2006.

[20] S. Lin, Y. Li, S.B. Kang, X. Tong, H.-Y. Shum. Diffusespecular separation and depth recovery from image sequences. In European Conference on Computer Vision, pages 210-224, 2002.

[21] I. Sato, Y. Sato, and K. Ikeuchi. Acquiring a Radiance Distribution to Superimpose Virtual Objects onto a Real Scene. IEEE Transactions on Visualization and Computer Graphics, 1999.

[22] R. T. Tan and K. Ikeuchi. Separating Reflection Components of Textured Surfaces using a Single Image. In International Conference on Computer Vision, pages 870-877, 2003.

[23] P. Thrift and C. Lee. Using highlights to constrain object size and location. IEEE Transactions on Systems, Man, and Cybernetics, 13:426-431, 1983.

[24] T. Yu, H. Wang, N. Ahuja, and W.-C. Chen. Sparse lumigraph relighting by illumination and reflectance estimation from multi-view images. In ACM SIGGRAPH, page 175, 2006.

[25] Y. Yu, P. E. Debevec, J. Malik, and T. Hawkins. Inverse global illumination: Recovering reflectance models of real scenes from photographs. In ACM SIGGRAPH, pages 215224, 1999.

[26] A. Zisserman, P. Giblin, and A. Blake. The Information Available to a Moving Observer from Specularities. Image and Vision Computing, 7(1):38-42, Feb. 1989. 